Revista de Psicología Vol. 32 (2), 2014 (ISSN 0254-9247)

\title{
Enfermedades médicas y depresión en el adulto mayor: características comunes y relación etiológica ${ }^{1}$
}

\author{
Hamer Bastidas-Bilbao ${ }^{2}$ \\ Universidad de los Andes, Colombia
}

\begin{abstract}
La revisión sistemática de 51 artículos en inglés publicados a partir del año 2000 en las bases de datos PsycNet, Ebsco y Science Direct, revela una alta comorbilidad de depresión en adultos mayores. Asimismo, se observa que los adultos mayores presentaban también enfermedad cerebrovascular, diabetes, Alzheimer y parkinsonismo, con sintomatología similar a la depresión debido al deterioro que provocan. El análisis permitió observar que estas enfermedades pueden anteceder a la depresión sin necesidad de un vínculo anatómicofuncional directo, por cuanto a las implicaciones psicosociales que conllevan. La depresión es también un factor de riesgo para la incidencia de enfermedad cerebrovascular y diabetes. Se recomienda la realización de más estudios longitudinales, con el fin de caracterizar pormenorizadamente el curso etiológico de la depresión.
\end{abstract}

Palabras claves: depresión, adulto mayor, enfermedades

Medical diseases and depression in older adults: common features and etiological relation A systematic review was conducted of 51 English-language papers published since 2000 in the following databases: PsycNet, Ebsco, and Science Direct. Findings reveal a high comorbidity of depression in older adults. Moreover, they also exhibit stroke, diabetes, Alzheimer's disease and Parkinsonism, with patterns of symptoms similar to those seen in depression resulting from disease impairment. Analysis shows that these diseases can also precede depression, even without an anatomical-functional link, due to the psychosocial burden borne by the patient. Depression is also seen as a risk factor for stroke and diabetes. Longitudinal studies are suggested to judiciously characterize etiological paths for depression more extensively.

Keywords: Depression, older adults, diseases

1 El autor agradece a Diana Agudelo Vélez, PhD., profesora del Departamento de Psicología de la Universidad de los Andes, Bogotá-Colombia, por sus comentarios en una versión preliminar de este artículo.

2 MSc. en Psicología Clínica y de la Salud, doctorando en Educación y asistente doctoral del Centro de Investigación y Formación en Educación (CIFE) / Universidad de los Andes, Bogotá-Colombia. Dirección postal: Carrera 1 No. 18 A - 12. Bloque Na. Of. 302. Contacto: hj.bastidas22@uniandes.edu.co 
La población de adultos mayores es menor al $9 \%$ del total de habitantes en países como Colombia, Ecuador, Perú y Venezuela (UNFPA $\&$ HelpAge International, 2012). En el caso de Colombia las personas mayores de 60 años constituyen el 8.9\% de la población, según el último censo nacional (Departamento Administrativo Nacional de Estadística, 2005). No obstante, a nivel global, la Organización Mundial de la Salud estima que la población de adultos mayores continuará creciendo hasta alcanzar los dos mil millones de adultos mayores hacia el año 2050 (Unsar \& Sut, 2010). Además, esta proyección señala que en Colombia, Ecuador, Perú y Venezuela, el total de adultos mayores se incrementará hasta alcanzar entre el $20 \%$ y el $24 \%$ de la población general (UNFPA \& HelpAge International, 2012).

Por otra parte, se estima que en el mundo más de 340 millones de personas han sufrido de depresión en algún momento de su vida (Egede \& Ellis, 2010) y, en el territorio colombiano, el estudio nacional de salud mental describe que los trastornos depresivos ocupan el segundo lugar en prevalencia, y más de la mitad de los adultos mayores han tenido su primer episodio depresivo después de los 60 años (Ministerio de la Protección Social, 2003). Adicionalmente, se estima que la depresión geriátrica se presenta en tasas del 1 al 5\%, y esta incidencia se incrementa junto con la edad. Más aún, el 13\% de la población geriátrica padece de síntomas depresivos clínicamente significativos que no cumplen los criterios diagnósticos de depresión mayor, ni depresión menor (Camus, Kraehenbühl, Preisig, Büla \& Waeber, 2004; Samaras, Rossi, Giannakopoulos \& Gold, 2010). Asimismo, la tasa de remisión de casos con depresión es baja y solo alcanza el 5\% del total de los casos detectados (Strober \& Arnett, 2009).

Las tasas de prevalencia y remisión deben ser analizadas detalladamente puesto que, en general, se ha considerado que los síntomas depresivos son un fenómeno normal en la población geriátrica (Polidori, 
Menculini, Senin \& Mecocci, 2001); no obstante, la depresión tiene serias implicaciones, provocando un incremento en las tasas de morbilidad, deterioro y ajuste social, que se traducen en mayor mortalidad. En este sentido, demandan gran atención los índices de suicidios en adultos mayores, los cuales han sido estimados en un $0.4 \%$, correspondientes a 15.10 por cada 100.000 habitantes (United States Centers for Disease Control and Prevention, 2010), siendo los más altos entre todos los segmentos etarios de la población estadounidense. Burke y Wengel (2003) señalan que los adultos mayores que cometen suicidio dan menos señales de alerta, usan métodos de mayor letalidad y lo hacen de forma más sistemática y decidida; sin embargo, incluso si se omite el suicidio como causa de muerte, se ha observado que la depresión en el adulto mayor se asocia a una mortalidad más temprana y a una mayor tasa de hospitalizaciones y costos de salud.

A la fecha, el diagnóstico de depresión se realiza siguiendo los criterios del DSM-V (American Psychiatric Association, 2013), los cuales incluyen algunos síntomas físicos tales como cambios psicomotores, perturbaciones en el apetito y el peso corporal, falta de energía, entre otros. Estos criterios resultan problemáticos cuando se emplean con adultos mayores debido a que ciertas enfermedades médicas también pueden enmascararse en síntomas similares a los de la depresión o viceversa, y por ello no es fácil establecer la fuente causal de los síntomas ni un diagnóstico único (Burke \& Wengel, 2003). De igual manera, se ha observado que ciertas enfermedades médicas tales como enfermedad de Parkinson, infarto, accidente cerebrovascular, trastornos endocrinos, diabetes, enfermedad pulmonar crónica, enfermedades infecciosas, etc. (Unsar \& Sut, 2010; Unützer, 2002), así como algunos medicamentos como las benzodiacepinas, los antihipertensivos, los anticancerígenos, entre otros, pueden causar depresión en momentos posteriores, o incrementar la severidad de síntomas depresivos ya presentes (Unützer, 2002). Es así como la depresión geriátrica se manifiesta de forma heterogénea debido a que su etiología solo puede explicarse recurriendo a diversos mecanismos patogénicos de orden biológico y psicosocial que coexisten en el individuo (Camus et al., 2004). Más aún, la relación 
entre la enfermedad física y la depresión no necesariamente es una relación directa, ya que la depresión puede desencadenarse a causa del malestar y la discapacidad provocada directamente por alguna enfermedad en particular o, incluso, por el deterioro físico y sensorial asociado al envejecimiento (Fiske, Wetherell \& Gatz, 2009; Unützer, 2002).

Esta vinculación entre la enfermedad médica y los síntomas depresivos tiene serias implicaciones, ya que el seguimiento irrestricto de los criterios del DSM-V (American Psychiatric Association, 2013) provocaría que múltiples pacientes no sean diagnosticados con depresión y, por lo tanto, no reciban atención especializada (Burke \& Wengel, 2003; Fiske et al., 2009). Por esta razón es importante identificar y comprender con mayor profundidad la relación existente entre las enfermedades médicas y la depresión, de tal manera que la fuente etiológica de los síntomas pueda estimarse con mayor precisión. Esto permite contrarrestar el posible sesgo de sobrediagnóstico de enfermedades médicas, que lleva a subdiagnosticar la depresión y, de igual forma, contribuye a que tampoco se pasen por alto síndromes físicos hasta el punto de incurrir en un sobrediagnóstico de la depresión (Fiske et al., 2009). Asimismo, si bien múltiples factores de riesgo de depresión tales como algunas variables demográficas, psicosociales o espirituales, no son susceptibles de intervención o modificación, lo cierto es que sí es posible intervenir, por lo menos de forma parcial, en algunas condiciones médicas, de forma que se pueda reducir el riesgo de aparición o agravamiento de la depresión (Lawhorne, 2005) y viceversa.

\section{Método}

La presente revisión sistemática se realizó a partir de estudios descriptivos o correlacionales, así como artículos de revisión teórica, encontrados en las bases de datos PsycNet, Ebsco y ScienceDirect, empleando los términos de búsqueda elder(ly), older adult(s), depression, late life depression con las siguientes variables: diabetes, Alzheimer, Parkinson, stroke. La búsqueda se realizó a nivel de título, resumen y 
palabras claves de los artículos, con el uso de conectores lógicos. Los artículos incluidos en el presente estudio presentaron las características siguientes: (a) estaban en idioma inglés, (b) fueron publicados a partir del año 2000, (c) constituían estudios descriptivos, relacionales, o revisiones teóricas, (d) los participantes eran adultos mayores, definido como aquel grupo de personas cuyas edades son iguales o superiores a 60 años (World Health Organization, 2014), (e) la temática estaba relacionada con la comorbilidad entre depresión y enfermedades médicas, la evaluación de factores de riesgo médicos para la depresión, y la estimación de la depresión como factor de riesgo para enfermedades médicas. Estos criterios de búsqueda permitieron la selección de un total de 51 artículos cuyo contenido fue analizado para la elaboración de esta revisión.

\section{Enfermedades cerebrovasculares y depresión}

En primer lugar, se observa una prevalencia significativa de depresión en adultos mayores que han sufrido accidentes cerebrovasculares (ACV). Esta prevalencia se estima de $11 \%$ a $50 \%$ durante el primer mes después del ACV, y solo disminuye de 1 a 2 años después (Camus et al., 2004; Graven et al., 2011; Gray et al., 2011; Hakim, 2011; Ostir, Berges, Ottenbacher \& Ottenbacher, 2011; Rao, 2000). El 25\% de los adultos mayores no muestra mejoría o incluso empeora (Ostir et al., 2011). Además, se observa mayor prevalencia de depresión en adultos mayores con demencia por ACV que en aquellos con enfermedad de Alzheimer (Rao, 2000). De esta manera, las enfermedades cerebrovasculares (ECV) y la depresión se asocian con un mayor riesgo de discapacidad (Licht-Strunk et al., 2004). Más aún, la depresión incrementa de forma significativa el riesgo de muerte en adultos mayores con diabetes, enfermedades cardiovasculares e hipertensión (Camus et al., 2004; Nuyen et al., 2007).

Sin embargo, a pesar de este elevado porcentaje de comorbilidad y sus implicaciones, la depresión puede pasar inadvertida, puesto que los profesionales de atención primaria suelen recurrir diferencialmente 
a explicaciones orgánicas de la sintomatología presente (Nuyen et al., 2007; Rao, 2000). Más aún, los perfiles sintomáticos de la depresión en adultos mayores con y sin ECV no se diferencian significativamente, excepto en su aparente duración (Luijendijk, Stricker, Hofman, Witteman \& Tiemeier, 2008), lo cual dificulta el diagnóstico diferencial y contribuye al sesgo de atribuir los síntomas de depresión exclusivamente a enfermedades orgánicas (Camus et al., 2004; Licht-Strunk et al., 2004). Esta situación se mantiene pese a que ciertos estudios continúan buscando evidencia a favor de definir la depresión vascular como una entidad específica dentro de los distintos síndromes depresivos de aparición en la vida tardía (Camus et al., 2004).

Adicionalmente, la comprensión del vínculo entre ECV y depresión es aún incipiente puesto que la mayoría de los estudios son de tipo transversal y no se cuenta con evidencia que permita identificar la direccionalidad de una posible relación causal entre la depresión y las enfermedades cerebrovasculares (Nuyen et al., 2007; Samaras et al., 2010), aunque se puede hablar de factores de riesgo asociados. Una de las posibles hipótesis para el estudio de la relación entre ECV y depresión señala que la depresión es un factor de riesgo o un factor etiológico antecedente de las ECV (Hakim, 2011; Kocer, Kocer, Degirmenci \& Eryılmaz, 2011; Rao, 2000). En este sentido, en la investigación realizada por Rao (2000) se señala que la depresión puede anteceder a la aparición de la ECV, ya que los adultos mayores deprimidos tienen una probabilidad 2.5 veces más elevada de padecer enfermedades subcorticales de la materia blanca y, a su vez, dichas enfermedades puede evolucionar hasta provocar accidentes cerebrovasculares.

Por otra parte, otros estudios sobre la relación entre depresión y ECV, presentan evidencia sobre una vía indirecta que involucra procesos patógenos como la enfermedad cardíaca o el infarto, así como procesos directos tales como hiperactividad simpático adrenal, inestabilidad ventricular y agregación plaquetaria, todos correlacionados con la depresión (Kocer et al., 2011). De esta forma, se ha descrito que la historia individual de la depresión o de los síntomas depresivos de larga duración en el adulto mayor se asocian de manera significativa con 
la aparición posterior de la enfermedad cardíaca isquémica e infarto al miocardio (Kocer et al., 2011), incluso hasta 10 años después del episodio depresivo (Camus et al., 2004; Luijendijk et al., 2008). Estos factores de riesgo cerebrovascular pueden duplicar el riesgo de ACV, sobre todo si se considera que están asociados a comportamientos de riesgo para la salud, tales como sedentarismo, consumo de sustancias y hábitos alimentarios deficientes (Camus et al., 2004; Luijendijk et al., 2008; Thomas, Kalaria \& O’Brien, 2004). Más aún, otros estudios han descrito a la depresión como precursora de las ECV debido al efecto vascular negativo provocado por algunos antidepresivos como los tricíclicos, o los inhibidores de la recaptación de serotonina, que modifican la función del endotelio arterial e inducen resistencia a la insulina, incrementando, en general, la predisposición de los pacientes hacia las enfermedades vasculares (Camus et al., 2004).

En segundo lugar, la hipótesis vascular o estructural, señala que la ECV es un factor etiológico en la depresión de aparición en la vida tardía (Bangen et al., 2010; Chau et al., 2010). Esta conceptualización implica que los factores de riesgo cerebrovascular, tales como hipertensión o enfermedad coronaria, están indirectamente relacionados con la aparición de la depresión puesto que, a lo largo del tiempo, contribuyen al desarrollo de enfermedades de los pequeños vasos cerebrales que perturba el funcionamiento neurobiológico provocando depresión (Bangen et al., 2010; Camus et al., 2004; Chatterjee, Fall \& Barer, 2010; Hakim, 2011; Nuyen et al., 2007; Samaras et al., 2010; Thomas et al., 2004). Estos hechos resultan especialmente relevantes para el adulto mayor ya que son ellos quienes están más expuestos a los distintos factores de riesgo cerebrovascular, y se ha hipotetizado que la relación entre factores de riesgo cardiovascular y depresión es más fuerte en adultos mayores con depresión de aparición tardía (Chatterjee et al., 2010; Nuyen et al., 2007), que en aquellos con depresión de aparición en edades más tempranas (Nuyen et al., 2007).

Asimismo, las lesiones subcorticales — vistas como hiperintensidades en las imágenes de resonancia magnética que son provocadas por cambios vasculares en el cerebro- se asocian a malos resultados en los 
tratamientos farmacológicos, así como a persistencia y mayor tasa de recidiva de la depresión (Camus et al., 2004; Lee \& Lyketsos, 2003; Licht-Strunk et al., 2004; Rao, 2000; Samaras et al., 2010; Strober \& Arnett, 2009; Thomas et al., 2004). Las hiperintensidades en los ganglios basales y en la sustancia blanca periventricular también se asocian al envejecimiento normal. Sin embargo, su mayor volumen se ha visto vinculado a la presencia de depresión geriátrica (Camus et al., 2004; Samaras et al., 2010). No obstante, a pesar de que la evidencia todavía no es concluyente, se cuenta con sustento empírico a favor de la asociación entre la disminución del flujo sanguíneo de las cortezas frontal y prefrontal bilaterales y la depresión. Incluso, en muestras comunitarias comprendidas por adultos mayores con depresión, se han encontrado mayores niveles de prevalencia de enfermedades cerebrovasculares, tanto sintomáticas como silenciosas (Camus et al., 2004; Rao, 2000). Se ha hallado también un cambio farmacodinámico manifestado en menores concentraciones de un metabolito de la serotonina -ácido 5-hidroxi-licético- en el fluido cerebroespinal en pacientes que experimentaron depresión tras el ACV, en comparación con participantes sin depresión y sin ACV (Rao, 2000).

En tercer lugar, se ha propuesto que las alteraciones en los mecanismos serotoninérgicos pueden ejercer una influencia simultánea que predisponga al individuo a desarrollar depresión y ECV (Rao, 2000). Por último, se ha planteado la hipótesis en torno a la depresión como una manifestación reactiva ante la ECV (Bangen et al., 2010; Chau et al., 2010; Graven et al., 2011; Rao, 2000). De esta manera, ciertas características asociadas a la ECV, tales como tiempo desde la aparición, tipo de lesión y grado de discapacidad provocado, pueden predisponer a la persona a reaccionar con sintomatología depresiva, cuya intensidad variará de acuerdo al curso de la enfermedad (Gray et al., 2011; Rao, 2000). Además, algunos factores psicosociales, como historia previa de depresión y el grado de apoyo social disponible, influyen en el curso de la depresión (Chau et al., 2010; Rao, 2000; Samaras et al., 2010). Finalmente, otras fuentes de evidencia proponen una variante de la hipótesis reactiva, señalando que la ECV puede ser un factor de vulnerabilidad 
y que el desencadenamiento efectivo de la depresión puede ser causado por un agente estresante de tipo psicosocial y de aparición posterior en el tiempo (Samaras et al., 2010).

\section{Diabetes y depresión}

A nivel mundial, se estima que más de 285 millones de adultos padecen de diabetes. En los países en desarrollo, la mayoría de diabéticos tienen entre 45 y 64 años de edad mientras que, en los países desarrollados, la mayoría de personas con diabetes son adultos mayores de 65 años (Egede \& Ellis, 2010). Se ha estimado que la prevalencia de depresión se duplica en adultos mayores que padecen de diabetes, en comparación con adultos mayores sanos (Bai, Chiou, Chang \& Lam, 2008; Camus et al., 2004; Khuwaja et al., 2010; Palinkas, Lee \& Barrett-Connor, 2004; Pawaskar, Anderson \& Balkrishnan, 2007; Shehatah et al., 2010). Algunos indicadores también señalan que la prevalencia de la depresión en esta población oscila entre el 3 y el $30 \%$ (Campayo et al., 2010; Maharaj, 2011; Pawaskar et al., 2007; Shehatah et-al., 2010), sumado a un 10\% de casos de depresión en pacientes diabéticos que no son diagnosticados (Egede \& Ellis, 2010). Por ello, se hace necesario identificar e intervenir apropiadamente en los casos de comorbilidad entre diabetes y depresión. Sin embargo, la labor de diagnóstico no es fácil, puesto que existe una superposición significativa entre los síntomas de la depresión y aquellos síntomas propios de un mal manejo de la diabetes, tales como fatiga, cambios en el peso corporal, cambios de apetito y perturbaciones de sueño (Shehatah et al., 2010).

La depresión en pacientes diabéticos se asocia a los peores resultados de tratamiento, menor funcionamiento físico, menor adherencia (Bellissimo et al., 2011), dificultades en el cumplimiento de los regímenes de ejercicio y alimentación (Camus et al., 2004), enfermedades vasculares, disfunción sexual, retinopatías, neuropatías y mayor mortalidad (Bai et al., 2008; Shehatah et al., 2010). Más aún, la depresión puede 
comprometer severamente el funcionamiento cotidiano del paciente (Bai et al., 2008; Pawaskar et al., 2007; Shehatah et al., 2010), y su curso puede ser crónico o intermitente (Shehatah et al., 2010). Asimismo, las investigaciones sobre la relación entre depresión y diabetes tienen diversas limitaciones metodológicas, tales como su naturaleza transversal (Palinkas et al., 2004), tamaños de muestra pequeños, ausencia de grupos de control y dificultades para diferenciar entre la diabetes de tipo 1 y la de tipo 2 (Shehatah et al., 2010). Son diversas las hipótesis que se han planteado para el estudio de la relación entre la depresión geriátrica y la diabetes. La primera de estas hipótesis señala que la depresión se desencadena debido a cambios bioquímicos propios de la diabetes tales como resistencia a la insulina (Khuwaja et al., 2010), cambios en las estructuras subcorticales y procesos inflamatorios que se asocian a cambios emocionales (Camus et al., 2004; Shehatah et al., 2010), así como alteraciones en el metabolismo de los neurotransmisores, las cuales han sido evidenciadas en modelos animales (Palinkas et al., 2004).

Otros estudios señalan que el riesgo de desarrollar depresión parece no ser mayor en los pacientes con diabetes una vez que se controlan las complicaciones y las enfermedades comórbidas (Luijendijk et al., 2008; Maharaj, 2011). Sin embargo, la evidencia respecto a la relación entre la diabetes y la probabilidad de la aparición de la depresión es aún contradictoria (Shehatah et al., 2010). Asimismo, el tipo de medicamentos antidiabéticos empleados se asocia con un incremento en el riesgo de la depresión en pacientes con diabetes tipo 2. Específicamente, el uso de antidiabéticos orales acarrea un menor riesgo de depresión en comparación con la terapia de insulina (Bai et al., 2008; Pawaskar et al., 2007). No obstante, este efecto se debe a que la farmacoterapia basada en antidiabéticos orales está diseñada para el tratamiento de diabetes de menor severidad que la terapia de insulina. En otras palabras, es el uso diferenciado de los fármacos, correspondiente a grados de severidad distintos, que conlleva mayor o menor carga psicológica requerida para el ajuste y cuidado de la enfermedad, el indicador de una variante de la hipótesis de depresión como reacción a la diabetes (Bai et al., 2008; Pawaskar et al., 2007). 
La segunda hipótesis señala que la depresión es precursora en la etiología de la diabetes. Estudios longitudinales muestran que la depresión mayor puede predecir la aparición de la diabetes en un lapso de 13 años. Sin embargo, esto no ocurre si se consideran formas menos severas de depresión u otros trastornos mentales (Palinkas et al., 2004). Adicionalmente, otros estudios señalan que la aparición de la diabetes tipo 2 parece ser independiente de la aparición de la depresión (Campayo et al., 2010). No obstante, este hecho no es tan claro en cuanto a la aparición de la diabetes tipo 1 (Luijendijk et al., 2008). A pesar de todo esto, se cuenta con evidencia que señala que los síntomas moderados o severos de depresión sí se relacionan con un incremento en el riesgo de incidencia de diabetes tipo 2 en un lapso de 5 a 10 años (Camus et al., 2004).

Más aún, algunos marcadores biológicos como los niveles de hemoglobina glucosilada HBA1C se han visto relacionados de forma significativa con el historial de depresión de aquellos pacientes con diabetes tipo 2 (Atlantis, Browning, Sims \& Kendig, 2010; Shehatah et al., 2010). De igual forma, se ha estimado que la depresión se asocia con un incremento del $60 \%$ en la probabilidad de riesgo de adquisición de diabetes tipo 2 (Atlantis et al., 2010; Shehatah et al., 2010). Otros mecanismos propuestos incluyen una activación del eje hipotalámico-pituitario-suprarrenal que, mediante elevaciones en los niveles de cortisol, puede antagonizar el proceso de eliminación de glucosa y contribuir a la acumulación de grasa visceral que fomenta la resistencia ante la insulina. Estos mecanismos también pueden estar acompañados por alteraciones en el sistema inmune — que pueden dañar las células pancreáticas-, así como por cambios conductuales asociados a malos hábitos de salud (Palinkas et al., 2004) que, sumado a otras respuestas fisiológicas asociadas a la depresión, tales como actividad hormonal contra-regulatoria, alteraciones en el transporte de la glucosa y activación inmuno-inflamatoria, pueden producir resistencia ante la insulina y alteraciones celulares que finalmente provoquen la aparición de la diabetes tipo 2 (Shehatah et al., 2010). 
En tercera instancia, se ha propuesto una hipótesis de la depresión como reacción emocional ante la enfermedad. Los síntomas depresivos pueden aparecer en respuesta al estrés psicosocial, así como a las demandas y limitaciones asociadas a la enfermedad y a su cuidado (Bai et al., 2008; Palinkas et al., 2004; Shehatah et al., 2010; Wexler et al., 2012). Sin embargo, es necesario considerar otras variables como el género, la educación y el nivel socioeconómico, que pueden facilitar o interferir en la aparición de la depresión (Pawaskar et al., 2007). Adicionalmente, la conceptualización de la depresión como reacción ante la enfermedad ha recibido apoyo empírico sustancial obtenido mediante la realización de estudios longitudinales que confirman el efecto sobre el estado del ánimo que tiene la diabetes tipo 2 como enfermedad crónica (Shehatah et al., 2010). A partir de esto, se ha observado que la mayor duración de la enfermedad y el mayor número de complicaciones, se asocian significativamente con mayores niveles de depresión (Bai et al., 2008; Bellissimo et al., 2011; Maharaj, 2011).

\section{Enfermedad de Alzheimer y depresión}

La enfermedad de Alzheimer es la causa más común de demencia en el adulto mayor, con tasas entre el 30 y el $70 \%$ de todos los casos de demencia (Strober \& Arnett, 2009; Winter, Korchounov, Zhukova \& Epifanova Bertschi, 2011; Yamane, Sakai \& Maeda, 2011). Además, se estima que la prevalencia de la depresión en pacientes con enfermedad de Alzheimer está entre el 10 y el 50\% (Heun, Kockler \& Ptok, 2002; Lee \& Lyketsos, 2003; Strober \& Arnett, 2009; Yamane et al., 2011). Sin embargo, hay estudios que estiman la prevalencia de este padecimiento en proporciones más elevadas, hasta aproximarse al 90\% (Even \& Weintraub, 2010; Strober \& Arnett, 2009; Winter et al., 2011). En particular, para el caso de la depresión mayor, la incidencia en adultos mayores con Alzheimer se calcula del 1 al 24\% (Heun, Kockler \& Ptok, 2003).

Existen síntomas comunes entre la depresión y la enfermedad de Alzheimer que dificultan el diagnóstico diferencial. Entre ellos 
se encuentran la pérdida de interés, la dificultad para pensar y concentrarse, y el enlentecimiento psicomotor, a lo cual que se suma la dificultad para diferenciar entre la depresión y la apatía típica de la demencia tipo Alzheimer (Engedal et al., 2011; Even \& Weintraub, 2010; Hattori, Yoshiyama, Miura \& Fujie, 2010; Lee \& Lyketsos, 2003; Mortby, Maercker \& Forstmeier, 2011; Winter et al., 2011). No obstante, también existen algunas diferencias en los patrones sintomáticos. Específicamente, los adultos mayores con depresión y enfermedad de Alzheimer reportan mayor número de perturbaciones del pensamiento (Engedal et al., 2011) e ideas de suicidio y culpa, mientras que aquellos que solo padecen depresión reportan ánimo negativo con mayor frecuencia (Lee \& Lyketsos, 2003). Sin embargo, esta diferenciación entre los patrones sintomáticos no ha podido ser replicada en todos los estudios (Heun et al., 2003; Strober \& Arnett, 2009). Pese a ello, existen otros síntomas, propios de la demencia tipo Alzheimer, independientemente de si hay coocurrencia de depresión, tales como la labilidad emocional, el insomnio y la incapacidad para expresar estados emocionales (Even \& Weintraub, 2010; Lee \& Lyketsos, 2003; Strober \& Arnett, 2009).

La diversidad sintomática resalta la importancia de que el clínico evalúe a profundidad los síntomas que pueden estar asociados a la depresión, puesto que el deterioro cognoscitivo también interfiere en la percepción y expresión de cambios en el estado de ánimo (Heun et al., 2003), especialmente si se considera que la afasia es un síntoma común en este tipo de demencia (Lee $\&$ Lyketsos, 2003). Además, la depresión en pacientes con enfermedad de Alzheimer se asocia a incrementos en la ideación suicida, menor calidad de vida, mayor discapacidad para la realización de actividades cotidianas, mayor mortalidad (Even \& Weintraub, 2010; Strober \& Arnett, 2009), así como aceleración en el deterioro cognoscitivo (Lee \& Lyketsos, 2003).

Como ya se mencionó anteriormente, una de las primeras hipótesis para el estudio de la relación entre la depresión geriátrica y las enfermedades médicas indica que la depresión puede ser una precursora en la etiología de la enfermedad de Alzheimer. En este sentido, se 
ha identificado que los adultos mayores con enfermedad de Alzheimer presentaron más síntomas depresivos a lo largo de sus vidas que los adultos mayores de la población general, por lo que se considera que la depresión puede constituirse como un factor (Heun et al., 2002, 2003; Reis, Brandão, Freire Coutinho, Engelhardt \& Laks, 2012) que podría duplicar el riesgo de aparición de la enfermedad de Alzheimer (Polidori et al., 2001). A su vez, se ha propuesto que la depresión de aparición en la vida tardía antecede causalmente a la aparición de la demencia tipo Alzheimer (Reis et al., 2012; Strober \& Arnett, 2009; Yan-Sheng, Meyer \& Thornby, 2001) en un período de 1 a 18 años (Even \& Weintraub, 2010; Lee \& Lyketsos, 2003).

En segundo lugar, otra hipótesis postula que el Alzheimer es el fundamento etiológico de la depresión. En estudios longitudinales se observó que las mayores tasas de incidencia de la depresión ocurren en un período de 5 años posterior a la aparición de la demencia (Heun et al., 2002). Es por ello plausible considerar que la depresión se relaciona con procesos neurobiológicos que son también responsables del deterioro cognoscitivo, tales como el deterioro del locus ceruleus y la sustancia negra (Heun et al., 2002, 2003), y el hipometabolismo (Caroli et al., 2010), de manera que la depresión puede llegar a considerarse como un síntoma inicial de los procesos degenerativos involucrados en la enfermedad de Alzheimer, más que como una señal de malestar psicológico. Por este motivo, se espera que los síntomas depresivos disminuyan a medida que el deterioro cognoscitivo progresa y dificulta percatarse de ellos (Heun et al., 2002, 2003; Strober \& Arnett, 2009).

Una tercera hipótesis señala que la depresión puede ser una manifestación reactiva ante la enfermedad de Alzheimer. En específico, se ha indicado que la depresión puede aparecer como una reacción emocional ante el progreso del deterioro cognoscitivo (Even \& Weintraub, 2010; Heun et al., 2002, 2003), viéndose influenciada por factores psicosociales como el género y la historia familiar e individual de depresión (Strober \& Arnett, 2009). Sin embargo, la evidencia no es concluyente, dado que en casos severos de demencia, no se observa una intensificación de los síntomas de depresión. Este hecho ha sido 
evaluado como evidencia en contra de la hipótesis de depresión como reacción emocional, debido al compromiso simultáneo entre el ánimo negativo, la percepción y la expresión de ese estado de ánimo, así como el deterioro de las funciones cognoscitivas (Lee \& Lyketsos, 2003; Strober $\&$ Arnett, 2009). Pese a ello, se estima que el $25 \%$ de pacientes con demencia tipo Alzheimer puede percibir apropiadamente su deterioro y con ello reaccionar de forma depresiva (Lee \& Lyketsos, 2003). Es así que la hipótesis reactiva debe tenerse en consideración cuando se prescribe la atención en salud necesaria para una porción significativa de adultos mayores con este tipo de demencia.

Finalmente, otros estudios proponen una cuarta hipótesis que recurre a procesos patológicos comunes, señalando que los síntomas de depresión y los déficits cognoscitivos observados en la enfermedad de Alzheimer son consecuencia de procesos neurodegenerativos. En este sentido, se ha postulado la existencia de factores genéticos que también son comunes a la depresión y a la demencia (Heun et al., 2002). Más aún, estudios post mortem han revelado una asociación entre la depresión y la pérdida de células noradrenérgicas en el locus ceruleus (Lee \& Lyketsos, 2003; Strober \& Arnett, 2009) y, por ello, esta nueva evidencia cambiaría significativamente el abordaje de la depresión y el Alzheimer, especialmente si los estudios posteriores confirman la existencia de mecanismos fisiopatológicos compartidos.

\section{Enfermedad de Parkinson y depresión}

La enfermedad de Parkinson se caracteriza por enlentecimiento motor, rigidez, temblores e inestabilidad postural. Además, es la segunda enfermedad neurodegenerativa más común tras la demencia tipo Alzheimer, y su mayor tasa de incidencia se presenta en adultos entre 55 y 66 años de edad (McDonald, Richard \& DeLong, 2003). Se ha estimado que entre el $25 \%$ y $50 \%$ de los pacientes con enfermedad de Parkinson pueden exhibir síntomas depresivos, los cuales contribuyen al deterioro funcional de los pacientes (Braam et al., 2010; 
Ehrt, Brønnick, Leentjens, Larsen \& Aarsland, 2006; McDonald et al., 2003; Stefanova et al., 2006; Zampieri \& Pedroso de Souza, 2011).

Los estudios prospectivos son en su mayoría escasos, y los reportes investigativos disponibles sufren de problemas metodológicos relacionados con el muestreo, el lugar de consecución de la muestra y los instrumentos usados para cuantificar la sintomatología depresiva (McDonald et al., 2003). A esto debe sumarse que el diagnóstico diferencial también es complejo puesto que los síntomas de la enfermedad de Parkinson se superponen a los síntomas de la depresión, en específico: dificultades de sueño, fatiga (Braam et al., 2010), enlentecimiento psicomotor, problemas de atención, función sexual disminuida, alejamiento de actividades sociales, ideación suicida (McDonald et al., 2003) y pérdida subjetiva de energía (Braam et al., 2010; Ehrt et al., 2006). Más aún, la apatía y las quejas cognoscitivas —atención, memoria, concentración - también pueden diagnosticarse de forma errada como depresión, cuando en realidad se han descrito como parte exclusiva del espectro sintomático de la enfermedad de Parkinson (Ehrt et al., 2006; McDonald et al., 2003).

Las implicancias de la depresión en pacientes con enfermedad de Parkinson se expresan en alteraciones de las habilidades motrices finas, el funcionamiento cognoscitivo, una influencia desfavorable en la calidad de vida, así como en un incremento en la mortalidad (Ehrt et al., 2006; McDonald et al., 2003). Asimismo, los síntomas depresivos son los más significativos para los pacientes y sus cuidadores (McDonald et al., 2003). Por esta razón, la depresión en los adultos mayores con parkinsonismo debe identificarse y tratarse apropiadamente en lugar de considerarse como un fenómeno normal del envejecimiento (Polidori et al., 2001) o como un simple componente del parkinsonismo. La relación entre enfermedad de Parkinson y depresión en adultos mayores también se guía por diversas hipótesis.

La primera hipótesis señala que la depresión en la enfermedad de Parkinson no es únicamente una reacción emocional al estrés psicosocial y a la discapacidad. Por el contrario, puede tratarse de una manifestación secundaria a la degeneración neuroanatómica de las vías 
corticales y subcorticales, así como de cambios en la función serotoninérgica y dopaminérgica (Ehrt et al., 2006; McDonald et al., 2003; Polidori et al., 2001). Adicionalmente, se ha observado que los pacientes con parkinsonismo padecen de degeneraciones en núcleos subcorticales en el área ventral-tegmental, hipotálamo, núcleo dorsal del rafé y locus ceruleus. Estas degeneraciones han sido observadas también en pacientes con depresión (Lieberman, 2006; McDonald et al., 2003). En este sentido, los estudios post mortem con neuroimágenes han revelado que los pacientes que padecieron simultáneamente de enfermedad de Parkinson y depresión tienen núcleos subcorticales más pequeños (McDonald et al., 2003). Más aún, el parkinsonismo en estadios iniciales puede afectar al locus ceruleus y a la amígdala, lo cual se asocia a la aparición de síntomas depresivos debido a los roles que estas estructuras tienen en el funcionamiento del sistema noradrenérgico (Braam et al., 2010).

En segundo lugar, al emplear una hipótesis centrada en la depresión como precursora, se ha señalado que esta puede preceder la aparición de la enfermedad de Parkinson durante un período de hasta 16 años (Lieberman, 2006). De esta manera, se ha encontrado evidencia de que la aparición de la depresión antes que los síntomas motores puede deberse a la expresión de un subtipo hereditario de Parkinson (Ehrt et al., 2006) hasta en un 30\% de los casos (Polidori et al., 2001). Sin embargo, aunque no se han identificado estudios que señalen que la depresión pueda contribuir etiológicamente a la aparición de la enfermedad de Parkinson, sí se ha observado que la depresión mayor puede señalar la aparición y la severidad de algunos síntomas cognoscitivos del Parkinson tales como demencia o psicosis. Por lo tanto, la depresión puede llevar a la ampliación del espectro sintomático más allá de lo motriz una vez que el parkinsonismo se encuentre presente (McDonald et al., 2003; Stefanova et al., 2006).

Por último, la hipótesis de la depresión reactiva también ha sido estudiada. Al respecto, claramente se observa que los pacientes con enfermedad de Parkinson y sus familias deben ajustarse al cuidado de una enfermedad crónica que puede tener implicaciones negativas a 
nivel laboral, familiar y social. A esto debe sumarse que los tratamientos son de tipo paliativo y su efectividad tiende a disminuir con el paso del tiempo. Todos estos elementos pueden provocar reacciones emocionales depresivas en los pacientes (Braam et al., 2010; McDonald et al., 2003) que también están moduladas por variables psicosociales.

\section{Discusión}

El contenido del análisis de los distintos artículos seleccionados para esta revisión puede agruparse de la siguiente manera:

a) Evidencia a favor de la hipótesis centrada en la depresión como factor etiológico precursor de las enfermedades médicas en adultos mayores: en el caso de las ECV se ha encontrado que la depresión, más que un factor etiológico, es un factor de riesgo que, a través de otros procesos fisiopatológicos —enfermedad cardíaca, infarto, entre otros - y ciertos cambios conductuales, predispone a que las personas eventualmente desarrollen alteraciones vasculares que desencadenen ACV. Para el caso de la diabetes, se han descrito mecanismos fisiológicos que desembocan en una resistencia a la insulina, los cuales son consistentes y brindan mayor relevancia al papel etiológico de la depresión. Finalmente, en el caso de las enfermedades neurodegenerativas como el Alzheimer y el parkinsonismo, se ha observado que la depresión es un factor de riesgo para la aparición de estas dos enfermedades aunque su papel etiológico no cuenta con el suficiente respaldo empírico.

b) Evidencia a favor de la hipótesis centrada en las enfermedades como factores etiológicos precursores de la depresión geriátrica: tanto las ECV, como la diabetes y las enfermedades neurodegenerativas - parkinsonismo y Alzheimer- demuestran ser sólidos precursores de la depresión, puesto que cada una de dichas enfermedades involucra el deterioro anatómico o funcional en distintas estructuras corticales y subcorticales, así como en diferentes sistemas neuroendocrinos. A su vez, estos deterioros o perturbaciones funcionales 
producen consistentemente cambios en el estado de ánimo que se corresponden con distintos tipos de síntomas de la depresión.

c) Evidencia a favor de la hipótesis centrada en la depresión geriátrica como reacción emocional ante las enfermedades médicas: esta es una de las hipótesis que cuenta con mayor evidencia empírica y hace referencia a que las implicaciones para la salud y para el funcionamiento individual y social de las personas con enfermedades crónicas son de tal magnitud que exceden los recursos disponibles por el individuo y su contexto hasta desencadenar síntomas depresivos. No obstante, es necesario mencionar que, aunque el fenómeno de la depresión reactiva ha sido observado repetidamente en enfermedades como la diabetes, el parkinsonismo y los ACV, su aparición no ha sido tan frecuente en la demencia tipo Alzheimer de mayor severidad. Esto significa que en los adultos mayores que se encuentran en los estados más avanzados de demencia, los síntomas de depresión tienden a desvanecerse debido a que la magnitud de las perturbaciones en las funciones cognoscitivas les impiden detectar cambios en su estado de ánimo, así como expresarlos de forma congruente.

Las fortalezas y debilidades de esta revisión son múltiples y ambas yacen en el tipo de artículos seleccionados. En primera instancia, se revisaron artículos monográficos, así como artículos empíricos centrados en el estudio de la relación entre las enfermedades médicas en mención y la depresión geriátrica. Esto se constituye como una ventaja puesto que permite emplear los hallazgos compilados en los artículos monográficos para usarlos a la hora de interpretar los artículos empíricos de tipo relacional. Asimismo, los autores de los artículos incorporados en esta revisión insisten en mencionar que las publicaciones sobre este tópico son aún escasas y heterogéneas, así que esta revisión permite incluir en un mismo lugar los hallazgos más recientes. Sin embargo, este hecho también se constituye como una debilidad puesto que la heterogeneidad de los artículos revisados impide el establecimiento de indicadores únicos que hayan sido empleados por todos los estudios 
analizados. De igual manera, la cantidad de artículos empíricos no es lo suficientemente vasta, y la variabilidad metodológica es alta, de forma que no es posible efectuar re-análisis de tipo cuantitativo.

Sin embargo, esta revisión sí permite observar que los estudios realizados hasta la fecha destacan que la depresión puede manifestarse de maneras relativamente similares tanto en la enfermedad de Alzheimer como en el parkinsonismo, lo cual ha sido explicado mediante procesos fisiopatológicos que son comunes para las dos enfermedades y que, a su vez, señalan la existencia de distintas fuentes etiológicas para la depresión, las cuales no necesariamente deben ser de tipo psicosocial, sino que también pueden ser de tipo biológico, tal como lo es en este caso.

Adicionalmente, este artículo permite reafirmar el impacto de las enfermedades crónicas en la vida y los roles sociales de los adultos mayores. Es así como distintas enfermedades pueden crear condiciones de vida que exceden los recursos de afrontamiento disponibles por los individuos y que actúan sobre factores previos de vulnerabilidad, que finalmente determinarán la aparición de síntomas o trastornos depresivos. De esta forma, es destacable que los artículos analizados señalen que, en aquellos casos en los que no se cuenta con evidencia del rol etiológico de la depresión, por lo menos sí se cuenta con evidencia de que la depresión es un factor que puede incrementar múltiples veces la probabilidad de riesgo de incidencia de cualquiera de las enfermedades aquí estudiadas, o complicar su sintomatología en caso de que las enfermedades ya estén presentes. Esto indica la importancia del diagnóstico y la intervención apropiada sobre los trastornos del estado de ánimo, debido al riesgo significativo que estas representan para la salud de los adultos mayores.

Para finalizar, esta revisión también permite observar la necesidad de realizar estudios longitudinales que faciliten la obtención de evidencia que vaya más allá de la descripción de la coexistencia de la depresión geriátrica con distintas enfermedades médicas, y mediante los cuales se pueda establecer un curso etiológico, así como la influencia de otras variables psicosociales y médicas que puedan tener un rol protector para la salud del adulto mayor. 


\section{Referencias}

American Psychiatric Association. (2013). Diagnostic and statistical manual of mental disorders (5th ed.). Washington, DC: American Psychiatric Publishing.

Atlantis, E., Browning, C., Sims, J. \& Kendig, H. (2010). Diabetes incidence associated with depression and antidepressants in the Melbourne longitudinal studies on healthy ageing (MELSHA). International Journal of Geriatric Psychiatry, 25(7), 688-696.

Bai, Y. L., Chiou, C. P., Chang, Y. Y. \& Lam, H. C. (2008). Correlates of depression in type 2 diabetic elderly patients: a correlational study. International Journal of Nursing Studies, 45(4), 571-579. http://dx.doi.org/10.1016/j.ijnurstu.2006.07.026

Bangen, K. J., Delano-Wood, L., Wierenga, C. E., McCauley, A., Jeste, D. V., Salmon, D. P. \& Bondi, M. W. (2010). Associations between stroke risk and cognition in normal aging and Alzheimer's disease with and without depression. International Journal of Geriatric Psychiatry, 25(2), 175-182.

Bellissimo, J. L., Holt, R. M., Maus, S. M., Marx, T. L., Schwartz, F. L. \& Shubrook, J. H. (2011). Impact of activity participation and depression on glycemic control in older adults with diabetes: glycemic control in nursing homes. Clinical Diabetes, 29(4), 139-144.

Braam, A. W., Beekman, A. T. F., Dewey, M. E., Delespaul, P. A. E. G., Fichter, M., Lobo, A. \& Copeland, J. R. M. (2010). Depression and parkinsonism in older Europeans: results from the EURODEP concerted action. International Journal of Geriatric Psychiatry, 25(7), 679-687.

Burke, W. J. \& Wengel, S. P. (2003). Late-life mood disorders. Clinics in Geriatric Medicine, 19(4), 777-797.

Campayo, A., de Jonge, P., Roy, J. F., Saz, P., de la Cámara, C., Quintanilla, M. A. \& Lobo, A. (2010). Depressive disorder and incident diabetes mellitus: the effect of characteristics of 
depression. American Journal of Psychiatry, 167(5), 580-589. http://dx.doi.org/10.1176/appi.ajp.2009.09010038

Camus, V., Kraehenbühl, H., Preisig, M., Büla, C. J. \& Waeber, G. (2004). Geriatric depression and vascular diseases: what are the links? Journal of Affective Disorders, 81(1), 1-16. http://dx.doi. org/10.1016/j.jad.2003.08.003

Caroli, A., Lorenzi, M., Geroldi, C., Nobili, F., Paghera, B., Bonetti, M. \& Frisoni, G. B. (2010). Metabolic compensation and depression in Alzheimer's disease. Dementia \& Geriatric Cognitive Disorders, 29(1), 37-45. http://dx.doi.org/10.1159/000257761 Chatterjee, K., Fall, S. \& Barer, D. (2010). Mood after stroke: a case control study of biochemical, neuro-imaging and socio-economic risk factors for major depression in strokesurvivors. $B M C$ Neurology, 10, 125-134. http://dx.doi.org/10.1186/1471-2377$10-125$

Chau, J. P. C., Thompson, D. R., Chang, A. M., Woo, J., Twinn, S., Cheung, S. K. \& Kwok, T. (2010). Depression among Chinese stroke survivors six months after discharge from a rehabilitation hospital. Journal of Clinical Nursing, 19(21/22), 3042-3050. http://dx.doi.org/10.1111/j.1365-2702.2010.03317.x

Departamento Administrativo Nacional de Estadística. (2005). Censo Nacional 2005. Bogotá: Autor.

Egede, L. E. \& Ellis, C. (2010). Diabetes and depression: global perspectives. Diabetes Research \& Clinical Practice, 87(3), 302-312. http://dx.doi.org/10.1016/j.diabres.2010.01.024

Ehrt, U., Brønnick, K., Leentjens, A. F. G., Larsen, J. P. \& Aarsland, D. (2006). Depressive symptom profile in Parkinson's disease: a comparison with depression in elderly patients without Parkinson's disease. International Journal of Geriatric Psychiatry, 21(3), 252-258. http://dx.doi.org/10.1002/gps.1456

Engedal, K., Barca, M. L., Laks, J. \& Selbaek, G. (2011). Depression in Alzheimer's disease: specificity of depressive symptoms using three different clinical criteria. International Journal of Geriatric Psychiatry, 26(9), 944-951. http://dx.doi.org/10.1002/gps.2631 
Even, C. \& Weintraub, D. (2010). Case for and against specificity of depression in Alzheimer's disease. Psychiatry \& Clinical Neurosciences, 64(4),358-366.http://dx.doi.org/10.1111/j.14401819.2010.02108.x

Fiske, A., Wetherell, J. L. \& Gatz, M. (2009). Depression in older adults. Annual Review of Clinical Psychology, 5, 363-389. http:// dx.doi.org/10.1146/annurev.clinpsy.032408.153621

Graven, C., Brock, K., Hill, K., Ames, D., Cotton, S. \& Joubert, L. (2011). From rehabilitation to recovery: protocol for a randomised controlled trial evaluating a goalbased intervention to reduce depression and facilitate participation post-stroke. BMC Neurology, 11(1), 73-82. http://dx.doi.org/10.1186/14712377-11-73

Gray, R. J., Myint, P. K., Elender, F., Barton, G., Pfeil, M., Price, G. \& Hardy, S. (2011). A depression recognition and treatment package for families living with stroke (DepReT-Stroke): study protocol for a randomised controlled trial. Trials, 12(1), 105112. http://dx.doi.org/10.1186/1745-6215-12-105

Hakim, A. M. (2011). Depression, strokes and dementia: new biological insights into an unfortunate pathway. Cardiovascular Psychiatry \& Neurology, 1-6. http://dx.doi.org/10.1155/2011/649629

Hattori, H., Yoshiyama, K., Miura, R. \& Fujie, S. (2010). Clinical psychological tests useful for differentiating depressive state with Alzheimer's disease from major depression of the elderly. Psychogeriatrics, 10(1), 29-33. http://dx.doi.org/10.1111/ j.1479-8301.2010.00308.x

Heun, R., Kockler, M. \& Ptok, U. (2002). Depression in Alzheimer's disease: is there a temporal relationship between the onset of depression and the onset of dementia? European Psychiatry, 17(5), 254.

Heun, R., Kockler, M. \& Ptok, U. (2003). Lifetime symptoms of depression in Alzheimer's disease. European Psychiatry, 18(2), 63. http://dx.doi.org/10.1016/S0924-9338(03)00003-8 
Khuwaja, A. K., Lalani, S., Dhanani, R., Azam, I. S., Rafique, G. \& White, F. (2010). Anxiety and depression among outpatients with type 2 diabetes: A multi-centre study of prevalence and associated factors. Diabetology \& Metabolic Syndrome, 2(1), 1-7. http://dx.doi.org/10.1186/1758-5996-2-72

Kocer, E., Kocer, A., Degirmenci, Y. \& Eryılmaz, M. (2011). Long-term depression is astrokeriskfactor. Acta Neuropsychiatrica, 23(6), 292296. http://dx.doi.org/10.1111/j.1601-5215.2011.00573.x

Lawhorne, L. (2005). Depression in the older adult. Primary Care: Clinics in Office Practice, 32(3), 777-792.

Lee, H. B. \& Lyketsos, C. G. (2003). Depression in Alzheimer's disease: heterogeneity and related issues. Biological Psychiatry, 54(3), 353. http://dx.doi.org/10.1016/S0006-3223(03)00543-2

Licht-Strunk, E., Bremmer, M. A., van Marwijk, H. W. J., Deeg, D. J. H., Hoogendijk, W. J. G., de Haan, M. \& Beekman, A. T. F. (2004). Depression in older persons with versus without vascular disease in the open population: similar depressive symptom patterns, more disability. Journal of Affective Disorders, 83(2/3), 155-160. http://dx.doi.org/10.1016/j.jad.2004.06.007

Lieberman, A. (2006). Are dementia and depression in Parkinson's disease related? Journal of the Neurological Sciences, 248(1-2), 138-142.

Luijendijk, H. J., Stricker, B. H., Hofman, A., Witteman, J. C. M. \& Tiemeier, H. (2008). Cerebrovascular risk factors and incident depression in community-dwelling elderly. Acta Psychiatrica Scandinavica, 118(2), 139-148.http://dx.doi.org/10.1111/j.16000447.2008.01189.x

Maharaj, R. G. (2011). Depression and type 2 diabetes mellitus: what we can learn from the Trinidad and Tobago experience, Editorial. Mental Health in Family Medicine, 133-136.

McDonald, W. M., Richard, I. H. \& DeLong, M. R. (2003). Prevalence, etiology, and treatment of depression in Parkinson's disease. Biological Psychiatry, 54(3), 363. http://dx.doi.org/10.1016/ S0006-3223(03)00530-4 
Ministerio de la Protección Social. (2003). Estudio Nacional de Salud Mental. Bogotá: Autor.

Mortby, M. E., Maercker, A. \& Forstmeier, S. (2011). Midlife motivational abilities predict apathy and depression in Alzheimer disease: the aging, demographics, and memory study. Journal of Geriatric Psychiatry \& Neurology, 24(3), 151-160. http://dx.doi. org/10.1177/0891988711409409

Nuyen, J., Spreeuwenberg, P. M., Beekman, A. T. F., Groenewegen, P. P., van den Bos, G. A. M. \& Schellevis, F. G. (2007). Cerebrovascular risk factors and subsequent depression in older general practice patients. Journal of Affective Disorders, 99(1-3), 73-81. http://dx.doi.org/10.1016/j.jad.2006.08.011

Ostir, G. V., Berges, I.-M., Ottenbacher, A. \& Ottenbacher, K. J. (2011). Patterns of change in depression after stroke. Journal of the American Geriatrics Society, 59(2), 314-320. http://dx.doi. org/10.1111/j.1532-5415.2010.03266.x

Palinkas, L. A., Lee, P. P. \& Barrett-Connor, E. (2004). A prospective study of type 2 diabetes and depressive symptoms in the elderly: the Rancho Bernardo study. Diabetic Medicine, 21(11), 11851191. http://dx.doi.org/10.1111/j.1464-5491.2004.01315.x

Pawaskar, M. D., Anderson, R. T. \& Balkrishnan, R. (2007). Selfreported predictors of depressive symptomatology in an elderly population with type 2 diabetes mellitus: a prospective cohort study. Health \& Quality of Life Outcomes, 5, 50-56. http:// dx.doi.org/10.1186/1477-7525-5-5

Polidori, M. C., Menculini, G., Senin, U. \& Mecocci, P. (2001). Dementia, depression and parkinsonism: a frequent association in the elderly. Journal of Alzheimer's Disease, 3(6), 553.

Rao, R. (2000). Cerebrovascular disease and late life depression: an age old association revisited. International Journal of Geriatric Psychiatry, 15(5), 419-433.

Reis, T., Brandão, C. O., Freire Coutinho, E. S., Engelhardt, E. \& Laks, J. (2012). Cerebrospinal fluid biomarkers in Alzheimer's disease and geriatric depression: preliminary findings from Brazil. CNS 
Neuroscience \& Therapeutics, 18(7), 524-529. http://dx.doi. org/10.1111/j.1755-5949.2012.00311.x

Samaras, N., Rossi, G., Giannakopoulos, P. \& Gold, G. (2010). Vascular depression. An age-related mood disorder. European Geriatric Medicine, 1(4), 220-225.

Shehatah, A., Rabie, M. A. \& Al-Shahry, A. (2010). Prevalence and correlates of depressive disorders in elderly with type 2 diabetes in primary health care settings. Journal of Affective Disorders, 123(13), 197-201. http://dx.doi.org/10.1016/j.jad.2009.09.002

Stefanova, E., Potrebic, A., Ziropadja, L., Maric, J., Ribaric, I. \& Kostic, V. S. (2006). Depression predicts the pattern of cognitive impairment in early Parkinson's disease. Journal of the Neurological Sciences, 248(1/2), 131-137. http://dx.doi.org/10.1016/j. jns.2006.05.031

Strober, L. B. \& Arnett, P. A. (2009). Assessment of depression in three medically ill, elderly populations: Alzheimer's disease, Parkinson's disease, and stroke. Clinical Neuropsychologist, 23(2), 205-230. http://dx.doi.org/10.1080/13854040802003299

Thomas, A. J., Kalaria, R. N. \& O’Brien, J. T. (2004). Depression and vascular disease: what is the relationship? Journal of Affective Disorders, 79(1-3), 81. http://dx.doi.org/10.1016/ S0165-0327(02)00349-X

United Nations Population Fund (UNFPA) \& HelpAge International. (2012). Ageing in the Twenty-First Century: A Celebration and a Challenge. Nueva York: Autor.

United States Centers for Disease Control and Prevention (CDC) (2010). Injury Prevention \& Control: Data \& Statistics. Recuperado febrero de 2014 desde: http://www.cdc.gov/injury/wisqars/ Unsar, S. \& Sut, N. (2010). Depression and health status in elderly hospitalized patients with chronic illness. Archives of Gerontology \& Geriatrics, 50(1), 6-10. http://dx.doi.org/10.1016/j.archger. 2008.12 .011

Unützer, J. (2002). Diagnosis and treatment of older adults with depression in primary care. Biological Psychiatry, 52(3), 285. 
Wexler, D. J., Porneala, B., Chang, Y., Huang, E. S., Huffman, J. C. \& Grant, R. W. (2012). Diabetes differentially affects depression and self-rated health by age in the U. S. Diabetes Care, 35(7), 1575-1577. http://dx.doi.org/10.2337/dc11-2266

World Health Organization (WHO). (2014). Definition of an Older or Elderly Person. Recuperado septiembre de 2014 desde http:// www.who.int/healthinfo/survey/ageingdefnolder/en/

Winter, Y., Korchounov, A., Zhukova, T. V. \& Epifanova Bertschi, N. (2011). Depression in elderly patients with Alzheimer dementia or vascular dementia and its influence on their quality of life. Journal of Neurosciences in Rural Practice, 2(1), 27-32. http:// dx.doi.org/10.4103/0976-3147.80087

Yamane, Y., Sakai, K. \& Maeda, K. (2011). Dementia with Lewy bodies is associated with higher scores on the geriatric depression scale than is Alzheimer's disease. Psychogeriatrics, 11(3), 157165. http://dx.doi.org/10.1111/j.1479-8301.2011.00368.x

Yan-Sheng, L., Meyer, J. S. \& Thornby, J. (2001). Longitudinal followup of depressive symptoms among normal versus cognitively impaired elderly. International Journal of Geriatric Psychiatry, 16(7), 718-727. http://dx.doi.org/10.1002/gps.423

Zampieri, M. \& Pedroso de Souza, E. A. (2011). Locus of control, depression, and quality of life in Parkinson's Disease. Journal of Health Psychology, 16(6), 980-987. http://dx.doi.org/ $10.1177 / 1359105310397220$

Recibido: 30 de junio, 2013 Aceptado: 30 de enero, 2014 